\title{
Latino Parents' and Adolescents' Perceptions of the Needs and Issues of Adolescents in Their Community
}

\author{
Diane de Anda $\cdot$ Todd M. Franke $\cdot$ Rosina M. Becerra
}

Published online: 2 June 2009

(C) The Author(s) 2009. This article is published with open access at Springerlink.com

\begin{abstract}
Data from The Community Needs Assessment Survey were examined to understand the issues parents and adolescents felt were most important to address for the adolescents in their community. The sample of 1,784 Latino respondents consisted of 892 parent/adolescent dyads. Factor analyses found parents and adolescents identified and prioritized the same six factors: education and career planning, abuse and victimization, adolescent behavior problems, adolescent sexuality, socioeconomic stressors, and relationships. However, parent ratings for all factors were higher, indicating a higher level of concern. The article analyzes differences by age/generation (parents versus adolescents), by immigrant versus native status, and by type of dyad based on the latter (i.e., US born adolescent/US born parent, US born adolescent/immigrant parent, immigrant adolescent/immigrant parent). The authors discuss the complex interaction of these factors and the implications for practice and research.
\end{abstract}

Keywords Latino adolescents - Needs assessment - Adolescent-parent dyads . Acculturation · Immigrant versus native status · Age/generational cohorts

\section{Introduction}

The overall growth of the US population documented in the 2000 Census includes a substantial increase in the youth population (ages 10-19) to 39.9 million, with 41.7 million projected in 2010 (US Census Bureau 2000). Moreover, the greatest increases were and will continue to be among youth of color, particularly Latino youth. Latino youth comprised $14 \%$ of the adolescent population in 2000 , but are

D. de Anda $(\bowtie)$. T. M. Franke · R. M. Becerra

Department of Social Welfare, School of Public Affairs, University of California, Los Angeles, 405 N. Hilgard Avenue, Los Angeles, CA 90095, USA

e-mail: ddeanda@ucla.edu 
projected to become $23 \%$ of the adolescent population by 2020 , with percentages significantly higher in the Western United States. Given the size of this population and the economic disadvantage experienced by a greater proportion of Latino families relative to other ethnic groups, it is important to develop an understanding of the needs of youth in Latino communities. Furthermore, the youth are not a homogeneous population, differing along many dimensions, the most notable being country of origin, with Latino youth and their families varying from recent immigrants to fourth and fifth generation US born citizens.

This study is a secondary data analysis examining perceptions of youth and their parents regarding the needs of the youth of a particular community to ascertain patterns of responses of various cohorts and adolescent-parent dyads and their implications for social work intervention in similar communities. Given that the median age of the Latino population is 26.6 years, that youth 19 years of age and under comprise more than a third of the Latino population (US Census Bureau 2000), and that the Latino population in Los Angeles is the largest Latino population in the United States, Los Angeles communities are ideal for this type of exploration.

\section{Background and Literature Review}

Needs of youth populations are generally determined and prioritized by examining population statistics. For example, the high dropout rate (44\%) of immigrant Latino youth (Llagas and Synder 2003), the Latina adolescent birth rate of 149.2 per 1,000 being the highest in the adolescent population (National Center for Health Statistics 2000), and a male homicide rate six times that of their White counterparts (National Center for Health Statistics, 2003) are often used to define areas for intervention focus, service provision, and program development. However, such a simplistic assessment of need does not account for the complex of factors that significantly impact the lives of youth and their families in these communities, many of which are not visible to the "outsider." Compounding social problems such as those identified above, are social, cultural, and economic factors. For example, a large percentage of Latino families must cope with economic stressors given that the median income for the Latino population is notably lower than that of the White non-Hispanic population (US Census Bureau 2008). Research has demonstrated that poverty and living in an economically disadvantaged neighborhood impact adolescent development and the relationship with primary agents of socialization, particularly with regard to mutual respect and social control (Elliot et al. 1996). Issues related to immigration status and related stressors exist between generations and among cohorts in the community; however, research into these areas has been extremely limited (Berry 1980; Buriel 1993; Camarillo 1990; Gil et al. 1994; Gonzales 1997; Lau et al. 2005; Partida 1996; Pasch et al. 2006; Ruiz 1996; Vega and Rumbaut 1991).

Early on, the research by Szapocznik et al. (1978) identified acculturation differences across generations, and further research documented problems and conflicts in the adolescent-parent dyad arising from acculturation gaps (Szapocznik and Kurtines 1993). In his descriptive study, Partida (1996) notes difficulties that 
arise as generations develop different rates and degrees of acculturation so that the younger members become "...the holders of power, knowledge, and control" ( $p$. 246). Moreover, Buriel's (1993) study of 317 Mexican American parents found significant differences in childrearing practices among parents of first, second, and third generation adolescents. The relationships between childrearing practices and generation were found to be complex: "across successive generations, Mexican American families undergo social and cultural changes that do not always conform to a linear model of acculturation... what is less obvious is how developmental processes within Mexican American families change across generations" (Buriel 1993). For example, fathers of first and third generation adolescents used controlling parenting styles more often, especially with their sons, than fathers of second generation adolescents. Recent research (Lau et al. 2005; Pasch et al. 2006) has called into question the assumption that adolescent-parent acculturation gaps are the primary source of familial conflict or youth behavior problems and suggested that factors beyond the acculturation gap need to be considered in understanding adolescent-parent interaction and family dynamics in Mexican American families.

Finally, parallel to the immigrants' acculturation experience, Latinos born in the United States experience a bicultural socialization process, in varying degrees being simultaneously socialized into the values, customs, norms, and mores of US mainstream and Latino cultures (de Anda 1984). This allows individuals to maintain their ethnic identity and culture while participating in mainstream society and its institutions. The bicultural balance probably varies considerably across individuals and generational cohorts. Given the complexity of acculturation, age and generational differences, and their interaction, it is important to explore how these factors impact the perception of social issues and problems most salient in the lives of the youth in these communities.

\section{Method}

Data were obtained from a closed-ended needs assessment measure that had been distributed to all 2510 ninth through twelfth grade students in an urban Los Angeles high school in Los Angeles County with a predominantly Latino population. The students completed the survey in homeroom and took a similar measure home for their parents, resulting in a return rate of $65.2 \%$ for the student and $47.0 \%$ for the parent surveys. Sample selection was based upon the respondent identifying as Latino (Mexican, Chicano, Central American, South American, or Other Latino) and indicating place of birth (US-yes or no). (Respondents from other ethnic groups each constituted less than one percent of the returned surveys.) All measures were completed anonymously, with each parent and adolescent measure pre-coded with the same number and A for adolescent or B for parent, so that the two could be matched. For the purposes of the secondary data analysis, only surveys with matched adolescent-parent dyads were included in the final sample. 


\section{Sample}

The final sample consisted of 1,784 respondents, constituting 892 adolescent-parent dyads. The majority $(n=654 ; 73.3 \%)$ of the adolescents were born in the US, in contrast to the parents, the majority $(n=774 ; 86.8 \%)$ of whom were immigrants, primarily from Mexico. A slightly higher percentage of females $(56.5 \%)$ than males $(43.5 \%)$ were represented in the adolescent sample. The mean age for the adolescents was 16.1 years, with little difference between the US born (15.9 years) and immigrant (16.1 years) respondents. Over two-thirds $(65.5 \%)$ of the adolescents were in the ninth $(35.3 \%)$ or tenth $(30.2 \%)$ grades, with small numbers in the eleventh $(19.9 \%)$ and twelfth (14.5\%) grades. The majority $(69.2 \%)$ of the immigrant adolescents had lived in the United States for 10 years or less, indicating that their early socialization experiences and part of their schooling occurred outside of the United States.

Females $(71.9 \%)$ greatly outnumbered males $(28.1 \%)$ in the parent sample, indicating that the majority of parents in the sample were Mexican immigrant mothers. The mean age for the parents was 41.9 years, 39.4 years for the US born parents and 42.3 years for the immigrant parents; however, this included an extremely wide range of ages, with the majority in the mid-thirties to early fifties. Hence, the parent sample is more clearly a generational cohort rather than an age cohort.

There were notable differences in the amount of schooling completed by US born and immigrant parents. The majority $(89.6 \%)$ of US born parents had completed some high school education or higher, while the majority of the immigrant parents had a sixth grade education or less, with only $34.1 \%$ having completed some high school or higher education. Only $33.3 \%$ of the parent sample had completed high school, with the high school completion rate the highest among US born parents, $61.5 \%$ in contrast to $21.1 \%$ for the immigrant parents.

Although no information regarding income was requested on the survey, the high school is located in an unincorporated city in Los Angeles County with a high poverty rate, $29.2 \%$ of the families below poverty level and $41 \%$ of the population receiving some form of public assistance (United Way of Greater Los 2007). Finally, the majority of the immigrant parents were not recent immigrants, having resided in the US an average of 19.5 years. Although only $5 \%$ were recent immigrants (5 years or less), over a quarter $(26.7 \%$ ) of the parents had immigrated to the United States subsequent to the birth of their child (15 years or less).

\section{The Measure}

The instrument, The Community Needs Assessment Survey, is a thirty-item measure on which high school students and their parents indicate their appraisal of the specific needs of the adolescents in their community. Staff at a long standing community social service agency were consulted to assure the comprehensiveness of the measure and that the format and phraseology were appropriate for the population. The instrument was pilot tested with a small number of students, who provided feedback regarding the clarity of the items and the instructions. 
The measure asks the respondent to evaluate thirty issues in reference to the following statement: "In your community, how important is it to deal with..." Responses are given along a five point Likert-type scale ranging from "very important" (5) to "not at all important" (1). The parent measure duplicates all items and response categories in the student survey, with both measures in an English/ Spanish bilingual format.

An exploratory factor analysis was conducted with the adolescent and parent samples. This provided a structural analysis of the measure by employing a factor extraction method with Varimax rotation to identify a consistent set of underlying concepts. To clearly separate the dimensions in the data, an orthogonal Varimax rotation method with loadings above 0.3 was accepted. Eigen values above 1, high item loading, and concept of "Simple Structure" (Thurstone 1954) served as criteria for factor selection. Tests for internal consistency were conducted on the items constituting each factor, with reliability coefficients ranging from 0.68 to 0.87 for adolescents and from 0.74 to 0.91 for parents. Ultimately, all remaining items were factor analyzed for both adolescents and parents, yielding six factors explaining $55 \%$ of the variance. The factor analyses conducted on both the parent and adolescent data yielded the same six factors. Conceptualized as six social issues, the factors that emerged were:

(1) adolescent sexuality (7 items), (2) relationships (5 items), (3) abuse and victimization (3 items), (4) socioeconomic stressors (6 items), (5) adolescent behavior problems (5 items), and (6) education and career planning (2 items). Table 1 presents the items in abbreviated form that comprise each of these factors.

\section{Data Analysis}

Two-one-way analysis of variance (ANOVA) procedures were conducted to ascertain if significant differences existed between adolescents (US born and immigrant) and parents (US born and immigrant) regarding the importance of the five community social issues. Additional calculations included strengths of association, which provided information regarding the correlation between the independent and dependent variables, and the effect size, which allowed an estimation of interpretive power. Differences in the rating of the various adolescent and parent groupings regarding the importance of the six community social issues (six factors) were examined via three match pairs $t$-tests. As the result of the use of multiple paired $t$-tests, the alpha level for all these tests was set to $p<0.01$, otherwise the nominal alpha was 0.05 .

\section{Findings}

The data were examined to explore differences based on age/generational cohort (adolescent vs. parent), country of origin (US born vs. immigrant), and dyadic combination of parent and adolescent (US born adolescent and US born parent, US born adolescent and immigrant parent, immigrant adolescent and immigrant parent). 
Table 1 Factor loadings

\begin{tabular}{|c|c|c|c|c|c|c|}
\hline & \multicolumn{6}{|l|}{ Factors } \\
\hline & 1 & 2 & 3 & 4 & 5 & 6 \\
\hline \multicolumn{7}{|l|}{ Adolescent sexuality } \\
\hline Teenage pregnancy & 0.694 & & & & & \\
\hline Birth control for teens & 0.676 & & & & & \\
\hline Teenage sexuality & 0.635 & & & & & \\
\hline Abortion & 0.570 & & & & & \\
\hline Homosexuality & 0.559 & & & & & \\
\hline Getting teens and parents talk about sex & 0.529 & & & & & \\
\hline Std's with HIV/AIDS & 0.481 & & & & & \\
\hline \multicolumn{7}{|l|}{ Relationships } \\
\hline Different groups of teens in community get along & & 0.722 & & & & \\
\hline People of different cultures, races, get along & & 0.683 & & & & \\
\hline Teen \& parents get along & & 0.607 & & & & \\
\hline Teen \& teachers get along & & 0.607 & & & & \\
\hline Teen boy/girlfriends get along & & 0.584 & & & & \\
\hline \multicolumn{7}{|l|}{ Abuse and Victimization } \\
\hline Physical abuse & & & 0.768 & & & \\
\hline Sexual abuse & & & 0.759 & & & \\
\hline Rape and date rape & & & 0.711 & & & \\
\hline \multicolumn{7}{|l|}{ Socioeconomic stressors } \\
\hline Problems having enough money for family needs & & & & 0.745 & & \\
\hline Unemployment & & & & 0.733 & & \\
\hline Medical care & & & & 0.663 & & \\
\hline Everyday stress and pressures & & & & 0.544 & & \\
\hline Lack of parent involvement in school & & & & 0.515 & & \\
\hline Parenting skills for teens & & & & 0.355 & & \\
\hline \multicolumn{7}{|l|}{ Behavior Problems } \\
\hline Graffiti/tagging & & & & & 0.659 & \\
\hline Violence in the schools & & & & & 0.629 & \\
\hline Students dropping out of school & & & & & 0.556 & \\
\hline Drugs and alcohol & & & & & 0.555 & \\
\hline Gangs & & & & & 0.543 & \\
\hline \multicolumn{7}{|l|}{ Education and career planning } \\
\hline Preparing for college & & & & & & 0.740 \\
\hline Preparing for jobs and careers & & & & & & 0.727 \\
\hline
\end{tabular}

Age/generation Comparisons: Adolescents and Parents

To determine if there were differences between age/generational cohorts in the degree of importance ascribed to each of the factors, $t$-test analyses were conducted on the means for the adolescent and parent samples. Statistically significant 
differences were found between parent and adolescent responses on five of the six factors (See Table 2).

A comparative examination of the means for the age/generational cohorts (See Fig. 1) reveals two consistent patterns: First, the prioritization of the factors is identical for the two groups. As indicated by the means, both samples rank the importance of the factors in the following order: (1) Factor 6, education and career planning, (2) Factor 3, abuse and victimization, (3) Factor 5, adolescent behavior problems, (4) Factor 1, adolescent sexuality, (5) Factor 4, socioeconomic stressors, (6) Factor 2, peer and adult relationships. The second consistent pattern that emerged from the data is the difference in the degree of concern between the two age/generational cohorts, with parents rating each factor of greater importance than the adolescents. All parent means are above 4.0 ("important") on the five point scale, with three of the six beyond the mid-point of 4.5, approaching "very important" (5.0). In contrast, only one of the adolescent ratings surpasses the 4.5 level, Factor 6 related to education and career preparation. The other means evidence a lesser degree of concern, with Factors 3 (abuse and victimization) and 5

Table 2 Paired $t$-tests age/generational cohort differences

\begin{tabular}{|c|c|c|c|c|}
\hline & $n$ & $M$ & $\mathrm{SD}$ & $t$ \\
\hline \multicolumn{5}{|c|}{ Adolescent sexuality } \\
\hline Parent & 828 & 4.30 & 0.75 & \\
\hline Adolescent & 828 & 3.91 & 0.80 & \\
\hline
\end{tabular}

Relationships

Parent

Adolescent

876

876

872

872

Adolescent

Socioeconomic stressors

Parent

Adolescent

848

848

856

856

Adolescent

Education and career planning

Parent

882

Adolescent

Behavior problems

$4.34 \quad 0.94$

$\begin{array}{llll}\text { Parent } & 856 & 4.53 & 0.65 \\ \text { Adolescent } & 856 & 4.11 & 0.77\end{array}$

$3.90 \quad 0.81$




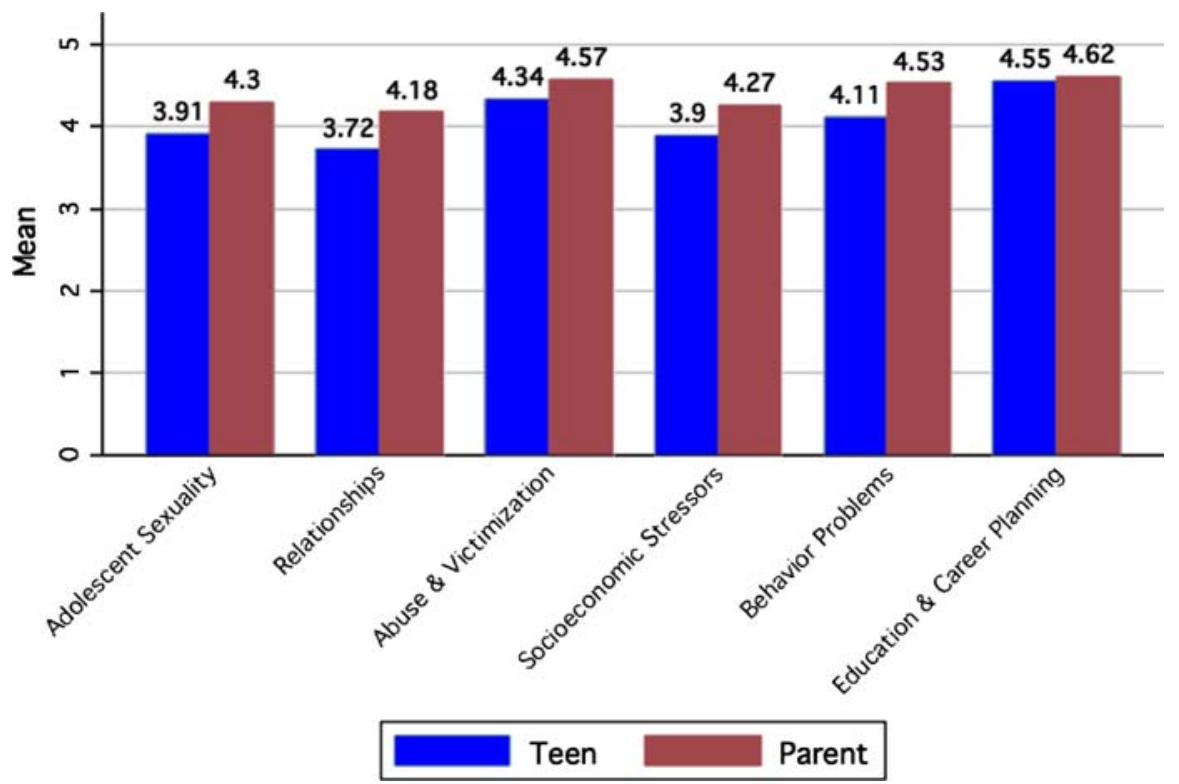

Fig. 1 Means of teen and parent factors

(adolescent behavior problems) rated "important," and the remaining three beyond the 3.5 midpoint approaching "important" (4.0). In other words, although both parents and adolescents agree on the rank order of the various factors in terms of their importance for adolescents in the community, the parents express a greater degree of urgency or concern with regard to these perceived needs and social issues.

\section{Country of Origin}

Figure 2 illustrates the complex interaction between age/generational cohort and country of origin. Note that the means are more similar for age/generational cohorts than for cohorts based on country of origin (US vs. immigrant). In other words, US born and immigrant adolescent ratings are more similar to each other than to parents with the same place of birth, and the case is the same for parents whose ratings are more similar to each other than to the adolescent group with the same place of birth. Although age/ generational cohort is preponderant, country of origin appears also to exert some influence, as both immigrant adolescents and immigrant parents tend to ascribe a greater degree of importance (evidenced by higher means) on every factor, with the exception of Factor 3 for the parents. Figure 2 clearly demonstrates the interaction of the influence of one's age/generational cohort and one's country of origin.

\section{Dyadic Comparisons}

Combining age/generation and country of origin, three adolescent/parent dyads were identified: US born adolescent/US born parent (US/US); US born adolescent/ 


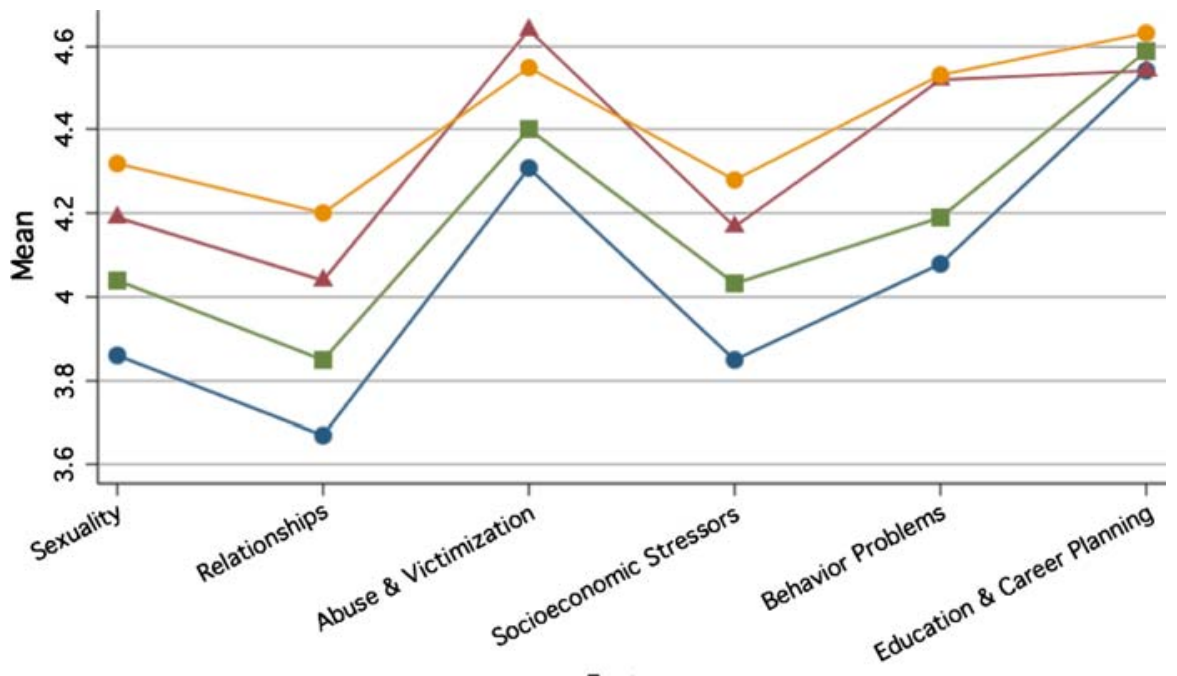

Factors

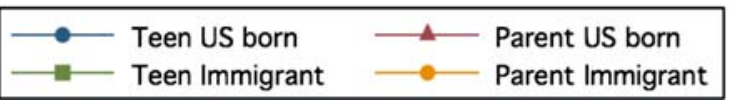

Fig. 2 Comparison by age/generation cohort

immigrant parent (US/IP); immigrant adolescent/immigrant parent (IA/IP). To determine if there were differences among these three groupings in the degree of importance ascribed to the various factors, univariate analyses of variance (ANOVAs) were performed on the dyadic data. Statistically significant differences were found on three of the six factors: Factor 1, adolescent sexuality, $F(2,826)=$ $4.309, p<0.05$; Factor 2 , peer and adult relationships, $F(2,874)=5.625$, $p<0.01$; and Factor 4, socioeconomic stressors, $F(2,846)=5.135, p<0.01$. Post hoc analyses indicated that the means for the IA/IP dyad were significantly higher than those of the other two dyads on Factors 1 and 2. On Factor 4, the difference was identified between the US/IP dyad and the IA/IP dyad. On all six factors, the means of the IA/IP dyad were the highest, indicating this dyad expressed the greatest concern regarding the social issues identified in the measure.

Based solely on the means, the above three factors were the lowest ranked of the six factors. The three factors, which evidenced no statistically significant differences among the three groupings, were ranked highest in importance and demonstrated a consensus among the groups in their prioritization. Factor 6, education and career planning, was viewed equally by the three dyads as of the greatest importance, with means of 9.10, 9.17, and 9.22 (US/US, US/IP, and IA/IP, respectively), followed by Factor 3, abuse and victimization, with means of $8.99,8.84$, and 9.01 , respectively, and by Factor 5, adolescent behavior problems, with means of 8.56, 8.62, and 8.77, respectively. Moreover, the consensus within the dyads is also greatest on Factor 6, with the difference in the means between adolescents and parents within each of the dyads between 0 and 0.08 , in contrast to the differences on the other factors ranging 
from 0.19 to 0.50 . Finally, the greatest amount of agreement (greatest similarity in means) between adolescents and parents within the dyads occurred in the IA/IP dyad and the least amount of agreement (most difference in the means) in the US/IP dyad. The combined difference in the means between adolescents and parents within the dyads for all six factors is as follows: US/IP $=2.04, \mathrm{US} / \mathrm{US}=1.84$, IA/ $\mathrm{IP}=1.71$. Inasmuch as the parent means were consistently higher than those of the adolescents across all of the dyads, paired t-test analyses were conducted on the means within each dyad across all six factors. The differences in the means between adolescents and parents were found to be statistically significant for every dyad on every factor with the exception of US/US and IA/IP on Factor 6 (See Table 3).

To determine if there were differences within the age/generational cohorts depending upon the adolescent/parent dyad, ANOVAs were conducted separately for the adolescents and parents with the comparison groups (independent variable) being composition of the dyad (US/US, US/IP, IA/IP). The results of the analysis of the adolescent data paralleled the findings from the preceding analysis of the dyadic data as a whole. Specifically, statistically significant differences were found between the adolescents in the various dyads on Factor 1 (adolescent sexuality), $F$ $(2,857)=4.424, p<0.05$; Factor 2 (relationships), $F(2,887)=4.645$; and Factor 4 (socioeconomic stressors), $F(2,866)=4.111, \mathrm{p}<0.05$. The ANOVAs of the parent data yielded statistical significance on only two of the factors: Factor 2, $F$ (2, $875)=3.620, p<0.05$; and Factor $4, F(2,867)=3.438, p<0.05$. The post hoc findings are not as straightforward as those in the previous dyadic analyses. For the adolescents, the pair-wise comparisons identified differences for Factors 1 and 4 to be between US born adolescents with immigrant parents and immigrant adolescents with immigrant parents. On the relationships factor (2), the difference was found between the immigrant adolescents and the US born adolescents irrespective of whether their parents were US born or immigrants. With regard to the parents, in the pair-wise comparisons, differences were found on Factor 2 between immigrant

Table 3 Paired $t$-test, adolescent versus parent within dyads by birthplace for factors 1-6

\begin{tabular}{|c|c|c|c|}
\hline Factors & US/US & US/IP & IA/IP \\
\hline \multirow[t]{2}{*}{ Adolescent sexuality } & $t=4.42 * * *$ & $t=10.87 * * *$ & $t=6.16^{* * *}$ \\
\hline & $d f=(110)$ & $d f=(498)$ & $d f=(217)$ \\
\hline \multirow[t]{2}{*}{ Relationships } & $t=5.44 * * *$ & $t=12.60 * * *$ & $t=7.60 * * *$ \\
\hline & $d f=(116)$ & $d f=(529)$ & $d f=(228)$ \\
\hline \multirow[t]{2}{*}{ Abuse and victimization } & $t=3.72 * * *$ & $t=5.11 * * *$ & $t=3.39 * * *$ \\
\hline & $d f=(115)$ & $d f=(524)$ & $d f=(230)$ \\
\hline \multirow[t]{2}{*}{ Socioeconomic Stressors } & $t=3.71 * * *$ & $t=9.67 * * *$ & $t=6.69 * * *$ \\
\hline & $d f=(113)$ & $d f=(509)$ & $d f=(223)$ \\
\hline \multirow[t]{2}{*}{ Behavior problems } & $t=7.15^{* * *}$ & $t=11.47 * * *$ & $t=7.62 * * *$ \\
\hline & $d f=(113)$ & $d f=(519)$ & $d f=(221)$ \\
\hline \multirow[t]{2}{*}{ Education and career planning } & $t=0.21$ & $t=2.65^{* *}$ & $t=0.83$ \\
\hline & $d f=(115)$ & $d f=(528)$ & $d f=(236)$ \\
\hline
\end{tabular}

$* * p<0.01 * * * p<0.001$ 
parents with US born adolescents and those with immigrant adolescents. The post hoc analysis of the parent data did not yield any significant differences in the three pair-wise comparisons for Factor 4.

\section{Discussion}

Although adolescents and parents differed in their perceptions of the degree of importance of the various social issues and problems, the extent of the consensus (ordering of the means) between the two generational cohorts is noteworthy. That is, parents and adolescents shared a common perception regarding the needs of the youth of the community. A contributing factor to this consensus may be the fact that this is an economically depressed community, which translates into very evident needs and poor resource availability.

The consistent pattern of significantly higher parental ratings is probably a function of developmental, experiential, and role differences. Adolescents are often prone to the "personal fable" (Elkind 1967), a perception of varying degrees of invulnerability that could reduce the level of concern over risk factors in their environment. In contrast, parents, due to their additional life experience with environmental risk factors and their caretaking role, could be expected to exhibit a greater level of concern. Since this was a survey of the respondents' perceptions, it is unclear whether the parents' or the adolescents' assessment of the urgency of intervention is more accurate in terms of the needs of the community. However, for the sake of program planning, there appears to be sufficient consensus with regard to areas in need of intervention.

It is noteworthy that both the adolescents and their parents identified education and career planning as the most important needs of the youth of the community, and that this was the case across all three of the dyads. The adolescents' concerns concur with de Anda et al.'s (1999; de Anda and Becerra 1997) findings in which high school students in the Los Angeles area reported that school related and future planning issues were the greatest stressors in their lives. This, along with parents' even higher means, contradicts stereotypic assumptions regarding the low educational aspirations of Latino populations. It appears that the respondents have high educational aspirations, but feel that supportive services are needed to achieve these educational goals. This may indicate a discrepancy between educational aspirations and educational expectations. The latter are dependent upon factors in the individual's environment, which increase the likelihood of attaining one's educational aspirations.

The importance given by parents to preparation for college despite the low educational achievement of the parent sample, particularly immigrant parents, suggests that the education level of the parents may be more a function of limited opportunities than cultural or individual values. The fact that the parents' means were higher than the adolescents' means testified to the value they place on education and their desire for their children to be offered educational opportunities. The higher level of importance ascribed to preparation for higher education by the immigrant adolescents and parents may be a reflection of the anxiety caused by a 
lack of familiarity with this area, particularly given the substantially lower level of education of the immigrant parent.

Both adolescents and parents identified victimization (physical abuse and sexual assault/abuse) to be second in importance, suggesting perceived high rates or high risk for the youth in their community. Although this is the only factor on which the US born parents expressed a greater degree of concern (See Fig. 2) than the immigrant parents, the high means for both were not found to be significantly different.

The third factor in priority, behavior problems, encompasses a very heterogeneous set of behaviors, all of which involve risk or loss and the potential for legal repercussions. Three (violence in the schools, drugs and alcohol abuse, and gangs) relate to physical danger and the remaining two (tagging and dropping out) to behavior that could have serious negative consequences. The statistically significant greater concern expressed by the parents may not only be due to developmental and role differences, but to differences in the proximity of the threat. Adolescents, who deal with the threat of violence on a daily basis, may be more able to assess their risk and the effectiveness of the various means they have developed to cope with this threat, while parents have only indirect knowledge and assess the threat based on the existence of risk factors in the environment, such as the presence of gangs. Or, in order to cope with the stress of a violent environment, the adolescents may minimize the assessment of personal risk to a greater extent than their parents. However, the adolescents' rating of "important" clearly indicates that they have not become desensitized to its impact on their lives.

When the sample is divided by age/generational cohort and immigrant status, a number of interesting patterns emerge. First, there is a marked similarity in the responses of the age/generational cohorts. As illustrated in Fig. 2, with the exception of Factor 6, the means for each factor are more similar based on age/ generation cohorts (US and immigrant adolescents versus US and immigrant parents) than based on country of origin (US born versus immigrants). For the adolescents, it appears that common socialization and developmental experiences result in the immigrant adolescents' perspectives not only mirroring those of their US born cohorts, but being more similar to these peers than to their parents' perspective. At the same time, the two parent groups appear more similar to each other than to their corresponding adolescent group. Common experiences in the role of parent in their community may help explain this similarity, particularly since the majority of immigrants had lived in the US between 16 and 25 years.

Clearly, age/generational cohorts perceive social issues and problems in similar ways and these differ from the perceptions of the other age/generational cohort. Elder (1980) argues for conducting research with attention to age and generational cohorts in the study of adolescent populations and their parents: "Generational differences in values may reflect the disparity in life stage between parents and offspring, since values are shaped by the imperatives of life situations, or they may indicate socialization differences that are linked to cohort membership and historical times" (p. 22). Each generational cohort shares a common set of "historical" experiences and experiences common to their life stage, both of which help shape the individual's perspective. The adults share the life stage of parenthood 
and its attendant responsibilities. The developmental processes of adolescence, particularly the primary developmental task of identity achievement (Erikson 1968), may serve as potent unifying experiences for the adolescent cohorts.

At the same time, there does appear to be an interaction of age/generation and the experience of being an immigrant, in that, with the exception of Factor 3 for the parents, the adolescent and parent immigrant groups ascribe higher levels of importance than their respective age/generational cohorts and demonstrate greater levels of agreement in their ratings. It is unclear whether this is the result of a shared experience as immigrants acculturating to new norms and values or early socialization to the culture of origin. However, the immigrant adolescents clearly demonstrate the effects of bicultural socialization (de Anda 1984), subscribing to the values translated, modeled, and reinforced by their US born peers, while simultaneously being influenced by the values and perspective of their culture of origin as modeled and reinforced by their parents, hence their "in between" position in Fig. 2. Perspectives regarding the importance of addressing social issues within the community are shaped by a complex of factors which include age, generation, and degree of socialization or acculturation to the society.

This study is unique not only in its pairing of the adolescent and his/her parent, but its further examination of the effect of the type of dyad based on immigrant or native status. Irrespective of whether the difference in the means among the groups reached statistical significance, the means were always highest for the IA/IP dyad on all six factors, indicating that the immigrant dyad experienced the highest level of concern. Moreover, there is a pattern in the means of the three groups across the factors that suggests the influence of acculturation: on all but Factor 3 (abuse and victimization), the order of the means from highest to lowest is IA/IP, US/IP, US/ US. The higher means of the immigrant parents probably account for some of this pattern, but the statistically significant differences between US and immigrant adolescents on three of the factors and the higher means for immigrant adolescents than their US cohorts on the remaining three also contributed to this pattern. Factor 3 is the exception, with a pattern of IA/IP, US/US, US/IP, because it is the only factor on which the US born parents achieved the highest mean. Because US adolescents had the lowest mean on Factor 3, pairing US adolescents with immigrant parents resulted in a lower score than that of the US/US dyad.

Another noteworthy pattern is the degree of congruence between adolescents and parents with the same country of origin. That is, IA/IP and US/US have a greater degree of congruence in their evaluations of importance than the US/IP dyad. Contributing factors may include exposure to similar or different socialization experiences, including socialization to cultural values and perspectives, and shared or unshared immigration and adjustment experiences. Given that the socialization experiences provided by the society at large, the educational system, and their peers have been substantially different for the US born adolescent and the immigrant parent, greater divergence in not unexpected. Because family socialization to a greater or lesser degree is to the culture of origin among families with immigrant parents, the extent to which the adolescent is bicultural may impact the degree of convergence or divergence in perceptions. Socialization to generation may be the most salient, however, since significant differences between adolescents and parents 
were found on almost every factor across every dyad. This difference, however, was only in degree, as both parents and adolescents rated the six factors to be important, differing only in the degree of perceived importance.

Note that significant differences were found among the three types of dyads on the three factors of lesser concern, but not those of highest priority. This suggests that dyadic dynamics are complex and may vary substantially based on the particular issue and its interaction with multiple factors influencing individuals, including the unique interplay of these factors and their consonance or dissonance within the specific dyad. Research that purports to explain or predict perceptions or behavior based on unidimensional models or simple interaction effects should be suspect.

\section{Implications}

Although the generalizability of the findings regarding specific areas of need are limited by the inability to determine how representative this sample is of other Latino communities, some of the patterns that emerged offer a number of implications for social work practice. For example, immigrant children and adolescents may appear to acculturate at a very rapid rate, soon resembling their US born cohorts. This acculturation process can be facilitated by actively employing these same cohorts to serve as models, mediators, and translators of cultural norms and common experiences (de Anda 1984). However, social workers should be aware that differences will still remain that serve as linkages to the parent culture. These linkages across generations are important to maintain, and the child or adolescent may need assistance in becoming truly bicultural, able to fit appropriately into the two cultural worlds so that he/she does not become "neither fish nor fowl," that is, seen as out of sync with both. Also vulnerable are the US born youth with immigrant parents given the discrepancies between parent and adolescent values and perceptions, a differential pattern of acculturation noted as early as 1978 by Szapocnik et al. in their work with Puerto Rican families. Although differences in perspectives between adolescents and their parents are to be expected, this study demonstrates that they may be primarily with respect to the degree of concern rather than the problem or issue itself. The role of the social worker would be to focus on common concerns in order to foster cross-generational understanding and cooperative efforts.

The findings suggest that immigrant groups may experience stressors in the environment more intensely than their US born cohorts, as the immigrant groups expressed greater urgency in dealing with the identified problems. This signals a need for interventions directed at providing resources, support, information, and skill building for this population to assist them in dealing with environmental stressors that tax their present coping skills or their preferred coping styles. Since coping styles are often culturally embedded, care must be taken to provide culturally appropriate alternatives.

The factor analysis demonstrates that resource poor communities are acutely aware of the needs of their community. The items in each of the factors share a 
common focus to which the respondents give a consistent response resulting in a pattern of priorities. This lends support to the principle of self-determination and the trend toward client collaboration. The findings further illustrate the potential for drawing inaccurate assumptions from a client's situation when the client's perceptions are not sought. For example, conclusions of low parental and student aspirations have often been drawn based on parental education level and the high dropout rates in the Latino community, leading to a focus on changing the attitudes and experiences of individuals that at times approached a "blame the victim" perspective instead of providing increased resources and opportunities. As early as 1981, de Anda's survey of Latina adolescents noted their high educational aspirations in contrast to their educational expectations. The primary importance given by both parents and adolescents to preparation for higher education directs service providers not only to acknowledge positive attitudes towards educational achievement, but to explore interventions that address community and societal barriers. This is particularly important in an atmosphere that is becoming hostile towards immigrant populations and may further limit opportunities. Successful intervention will need to include supports for first generation college students adjusting to the demands of the unfamiliar college environment.

The findings also demonstrate the importance of recognizing the individual, whether a client or a research participant, as multidimensional (Falicov 1995), experiencing socialization forces from more than one source. The fact that socialization to one's age/generation cohort appeared more potent than socialization to the culture of one's country of origin is particularly noteworthy and warrants further research, particularly in communities with large immigrant populations with immigrant parents and US born children. Moreover, one's perception is a complex interaction of multiple experiences, as patterns reflecting both generation and immigrant versus non-immigrant status did emerge. These differences become particularly important when attempting to understand and intervene with adolescent-parent dyads.

The findings suggest that in developing and planning programs, agencies should not only begin with needs assessment to ascertain the views of potential clients, but examine different perceptions of need across a number of factors, including ethnic group, age and generational cohort, country of origin, and acculturation level, and the impact of these differences on family and community dynamics. One should not assume that needs or views and priorities regarding services are homogeneous within the community, but acknowledge that programs may need to be adapted for various groups within the populations served. Social work agencies must recognize that a complex of factors affect the perception of need and find a means to determine which are the most salient in their community.

Acknowledgments Survey funded by a Community Challenge Grant, California Department of Health Services, Office of Family Planning, Grant No. 05-45275.

Open Access This article is distributed under the terms of the Creative Commons Attribution Noncommercial License which permits any noncommercial use, distribution, and reproduction in any medium, provided the original author(s) and source are credited. 


\section{References}

Berry, J. W. (1980). Acculturation: As varieties of adaptation. In A. M. Padilla (Ed.), Acculturation: Theory models, and some new findings (pp. 9-26). Boulder: Westview Press.

Buriel, R. (1993). Childrearing orientations in Mexican American families: The influence of generation and sociocultural factors. Journal of Marriage and the Family, 55(4), 987-1000.

Camarillo, A. (1990). Chicanos in California: A history of Mexican Americans in California. In. Sparks: Materials For Today's Learning.

de Anda, D. (1981). Developing a diagnostic profile of Chicana students. Social Work in Education, 3, 5-21.

de Anda, D. (1984). Bicultural socialization: Factors affecting the minority experience. Social Work, 29(2), 101-107.

de Anda, D., Baroni, S., Boskin, L., Buchwald, L., Morgan, J., \& Ow, J., et al. (1999). Stress, stressors and coping among high school students. Children and Youth Services Review, 21.

de Anda, D., and Becerra, R. M. (1997). The community needs assessment survey. Unpublished manuscript.

Elkind, D. (1967). Egocentrism in adolescence. Child Development, 38(4), 1025-1034.

Elliot, D. S., Wilson, W. J., Huizinga, D., Sampson, R. J., Elliott, A., \& Rankin, B. (1996). The effects of neighborhood disadvantage on adolescent development. Journal of Research in Crime \& Delinquency, 33(4), 389-426.

Falicov, C. J. (1995). Training to think culturally: A multidimensional comparative framework. Family Process, 34, 373-388.

Gil, A. G., Vega, W. A., \& Dimas, J. M. (1994). Acculturative stress and personal adjustment among Hispanic adolescent boys. Journal of Community Psychology, 22, 43-53.

Gonzales, G. M. (1997). The emergence of Chicanos in the twenty-first century: Implications for counseling, research, and policy. Journal of Multicultural Counseling \& Development, 25(2), 94-106.

Lau, A. S., Mc Cabe, K. M., Yeh, M., Garland, A. F., Wood, P. A., \& Hough, R. L. (2005). The acculturation gap-distress hypothesis among high risk Mexican American. Journal of Family Psychology, 19(3), 367-375.

Llagas, C., \& Synder, T. (2003). Status and trends in the education of hispanics. Retrieved. from.

National Center for Health Statistics. (2000). Table DP-1.

National Center for Health Statistics. (2003). Table 2 homicide percentages and rates by race/ethnicity and gender ages 15-24. National Vital Statistics Reports, 52(9).

Partida, J. (1996). The effects of immigration on children in the Mexican-American community. Child \& Adolescent Social Work Journal, 13(3), 214-241.

Pasch, L. A., Deardorff, J., Tschann, J. M., Flores, E., Penilla, C., \& Pantoja, P. (2006). Acculturation, parent-adolescent conflict, and adolescent adjustment in Mexican American families. Family Process, 45(1), 75-86.

Ruiz, V. L. (1996). "Star Struck": Acculturation, adolescence, and the Mexican-American woman, 19201950. In D. G. Gutierrez (Ed.), Between two worlds: Mexican immigrants in the United States (Vol. 15, pp. 125-147). Wilmington: Scholarly Resources.

Szapocznik, J., \& Kurtines, W. M. (1993). Family psychology and cultural diversity: Opportunities for theory, research, and application. American Psychologist, 48, 400-407.

Szapocznik, J., Scopetta, M. A., Kurtines, W. M., \& Arnalde, M. A. (1978). Theory and measurement of acculturation. Interamerican Journal of Psychology, 12, 113-130.

Thurstone, L. L. (1954). An analytical method for simple structure. Psychometrika, 19, 173-194.

United Way of Greater Los Angeles. (2007). Zip code databook for los angeles county service planning area 7-east. Los Angeles, CA: Center for Community Research and Solutions.

US Census Bureau. (2000). Profile of general demographic characteristics of the United States.

US Census Bureau. (2008). DeNavas-Walt, Carmen, Proctor, Bernadette, D., \& Smith, Jessica, C. US Census Bureau, Current Population Reports, P60-235, Income, poverty, and health insurance coverage in the United States: 2007. US Government Printing Office, Washington, DC.

Vega, W. A., \& Rumbaut, R. G. (1991). Ethnic minorities and mental health. Annual Review of Sociology, $17,351-383$. 